\title{
LINEAR VERSUS NONLINEAR ALLOCATION RULES IN RISK SHARING UNDER FINANCIAL FAIRNESS
}

BY

JOHANNES M. SCHUMACHER

\begin{abstract}
In a risk exchange, participants trade a privately owned risk for a share in a pool. If participants agree on a valuation rule, it can be decided whether or not, according to the given rule, these trades take place at equal value. If equality of values holds for all participants, then the exchange is said to be "financially fair". It has been shown by Bühlmann and Jewell (1979) that, under mild assumptions, the constraint of financial fairness singles out a unique solution among the set of all Pareto efficient risk exchanges. In this paper, we find that an analogous statement is true if we limit ourselves to linear exchanges. Conditions are provided for existence and uniqueness of linear sharing rules that are both financially fair and Pareto efficient among all linear sharing rules. The performance of the linear rule is compared to that of the general (nonlinear) rule in a number of specific cases.
\end{abstract}

\section{KEYWORDS}

Risk sharing, allocation rules, financial fairness, Borch theorem, syndicates.

\section{INTRODUCTION}

Pooling of risks is a classical topic in actuarial science. A discussion of the early contributions of de Finetti and Borch is given by Seal (1969), and a textbook treatment is available for instance in Rotar (2007, Ch. 12). Research interest in recent years has focused in particular on efficient risk sharing between agents whose preferences are described by monetary valuation functionals (Chateauneuf et al., 2000; Barrieu and El Karoui, 2005; Filipovic and Svindland, 2008; Jouini et al., 2008).

A specific motivation for the present paper is the sharing of investment risks within a collective pension fund. Risk sharing agreements embedded in pension schemes in various countries, and in particular in the Netherlands, have been surveyed by Chen and Beetsma (2015). The typical Dutch occupational 
pension scheme may be described briefly as follows. Participants pay contributions during their working life, and receive benefits after retirement in the form of an indexed annuity. The fund needs to meet solvency requirements, and therefore the level of indexation that is applied in a given year depends, via the funding ratio, on realized investment returns as well as on other economic indicators such as interest rates. Because indexation is applied to accrued rights as well as to benefits, participants who are not yet retired take a share in risk bearing. The question arises what would be a reasonable way of allocating the investment risk, given different attitudes toward risk among the participants. For instance, indexation could be made age-dependent, as discussed by Molenaar and Ponds (2012/13).

In a discussion of risk sharing agreements within a collective, attention should be paid to the possible presence of cross-subsidies between (groups of) participants. Here, one needs to distinguish between transfers ex ante and transfers ex post. It is in the nature of risk sharing that, after resolution of uncertainty, transfers between agents may take place in the sense that agents receive amounts that are different from their early expectations. Before uncertainty is resolved, the situation of participants in an investment collective may be described by saying that they hold contingent claims that are specified by the risk sharing agreement, and which they have acquired on the basis of the contributions they have paid. According to the principle of absence of arbitrage (and assuming no subsidies from outside, nor any draining), the net present value of the investment pool must be equal to the sum of the contributions brought in by the participants, and it must also be equal to the sum of the net present values of the contingent claims held by the participants. The equality of the sums however does not imply equality between the value of the contribution and the value of the claim for each participant individually. If, for some partipants, this equality does not hold, then the risk sharing system effectively generates ex ante transfers of value between agents. The notion of financial fairness that is used in this paper comes down to the requirement of absence of such transfers.

As a simple example of the type of situation that is of interest in the present paper, consider the following. Two investors are owners of projects in which they have invested a certain amount of money and which will generate an uncertain return at a given moment in the future. The investors may choose to pool the projects, which means that they will share the total revenue according to a certain rule which is determined in advance. For instance, they may agree to take a fixed percentage each, corresponding to the amounts that they invested; this is a financially fair rule, which will be referred to as the proportional rule. The agents may also agree to a somewhat more elaborate scheme, in which they each receive a fixed amount plus a fixed percentage of the amount (possibly negative) that remains after the fixed amounts have been subtracted from the actually realized revenue. This is called a linear allocation rule. Proportional rules are completely determined by the constraint of financial fairness, but linear rules are not; the remaining degrees of freedom can be used to find agreements that are relatively 
more suitable given possible differences in risk appetite between the two agents. Finally, the agents may also use a nonlinear rule in which the amounts allocated to the agents are determined as general (i.e., not necessarily linear) functions of the realized revenue. In Section 4 of this paper, several situations of the above type will be analyzed, featuring various specifications of the preferences of the two investors and various degrees of correlation between the two investment projects.

The risk sharing contracts that we analyze here are supposed to be concluded between agents who have access to the same information, so that we do not have a situation of the principal/agent type, and neither do we consider complications arising from moral hazard. It may furthermore be noted that the notion of financial fairness that we use is different from notions of fairness typically used in the literature on fair division (see for instance Brams and Taylor, 1996). Financial fairness is a time-indexed notion, since it is based on net present values. In this paper, we only consider single-period models. Multiperiod risk sharing problems under financial fairness are discussed by Bao et al. (2017).

We use the term "risk" in this paper to refer to an uncertain monetary outcome, without necessarily a negative connotation. Given the motivation of the paper as a study of pooled investments, positive values of the random outcome are interpreted as gains, rather than as losses. The results of the paper can be applied, mutatis mutandis, to pooling of underwriting risk between insurance companies.

To define financial fairness, we need a valuation operator; moreover, the net present values of agents' stakes must be available. Furthermore, information on agents' preferences is required to define Pareto efficiency. For the purpose of valuation, we assume that a valuation operator is given in the usual form of a risk-neutral measure. Preferences are taken to be of the traditional von Neumann-Morgenstern type (expected utility).

The perspective in this paper is essentially that of a social planner who fixes, possibly on the basis of consultations of the participants in the collective, all ingredients of the risk sharing situation such as the agents' utility functions and the pricing measure. Agents are modeled as price-takers. Risk sharing with endogenous prices leads to the notion of general equilibrium; the use of exogenous prices, as applied here, can be considered appropriate for the analysis of collectives that form only a small part of the general economy.

Collectives that operate under financial fairness might be called "fixedvalue" syndicates, as opposed to the "fixed-weight" syndicates that are the main object of study in the classical paper by Wilson (1968). In Wilson's paper, a particular Pareto efficient solution is selected by assigning weights to agents, so that a combined utility function can be formed; these weights are assumed to be fixed. Under financial fairness, particular Pareto solutions are singled out on the basis of claim values (ownership rights) that are associated to the agents; weights are determined implicitly, as a function of the claim values. 
An allocation rule is said to be a Pareto efficient and financially fair (PEFF) solution if it is financially fair, and Pareto efficient even among allocation rules that are possibly not financially fair. If we would be looking for risk sharing contracts that are Pareto efficient within the class of financially fair solutions, then uniqueness in general cannot be expected. However, the notion of a PEFF solution is based on the stronger requirement that Pareto efficiency should hold regardless of financial fairness. Existence and uniqueness of PEFF solutions for collectives of expected-utility agents was proved, under mild assumptions, by Bühlmann and Jewell (1979), who made use of an idea by Gale (1977) (see also Gale and Sobel, 1979). An efficient algorithm for computing PEFF solutions was proposed by Pazdera et al. (2017). The proof of convergence of the algorithm in this paper is based on nonlinear Perron-Frobenius theory, and leads to an existence and uniqueness proof for PEFF solutions that is different from the one provided by Bühlmann and Jewell (1979).

Linear allocation rules, ${ }^{1}$ as already defined above, are quite common in practice. For von Neumann-Morgenstern agents, a necessary and sufficient condition for a linear allocation rule to be Pareto efficient among all allocation rules is that, subject to the linear rule, the agents should all have the same cautiousness with respect to the pooled risk (Huang and Litzenberger, 1985). ${ }^{2}$ This condition is satisfied when the agents in the collective have identical preferences and the allocation is purely proportional, when the agents all have constant absolute risk aversion, and when they all have constant relative risk aversion with the same risk aversion coefficient. In other cases, however, such as the case of power utility agents with different risk aversion coefficients, the condition is typically not satisfied, which means that linear allocation rules are not Pareto efficient.

A weaker notion of Pareto efficiency that may be applied to linear allocation rules is efficiency within the restricted class of all linear rules. In this paper, we answer the question whether within this class there is an analogous result to the theorem proved by Bühlmann and Jewell (1979) on the uniqueness of PEFF allocation rules. In other words, within the class of linear allocation rules that are Pareto efficient in the weaker sense, does financial fairness single out a unique solution? Sufficient conditions are provided below under which the answer to this question is positive. While these conditions are somewhat more restrictive than in the analogous case of general (possibly nonlinear) allocation rules, they are still satisfied for a broad class of utility functions.

This theoretical result leads to a uniquely determined linear allocation rule for a wide range of risk sharing situations. The performance of this linear rule (the linPEFF solution) may be compared to the performance of the nonlinear rule that can be derived from an application of Pareto efficiency and financial fairness in the fully nonlinear context (the general PEFF solution). In a second part of the paper, we carry out computations for a few particular cases in order to obtain an indication of the circumstances under which there are substantial advantages to working with a nonlinear rule rather than with the simpler linear rule. 
The paper is organized as follows. Following this introduction, there is a section in which the problem is formulated in mathematical terms. The main theoretical result follows in Section 3. Section 4 presents a comparison of the performance of the linPEFF rule to the general (nonlinear) PEFF rule in a number of special cases. Conclusions follow in Section 5. There is an appendix containing some additional material and most of the proofs.

\section{Problem SETting}

We consider a collective consisting of $n$ agents. The collective is owner of a risk $X$, which is defined as a random variable on a measurable space $(\Omega, \mathcal{F})$. On this space, we work with two measures, which are denoted by $P$ and $Q$. The measure $P$ is the "real-world" measure that is used by the agents to evaluate expected utility. The measure $Q$ serves to define a notion of "financial fairness". Both measures are exogenously given and are not agent dependent. The measure $Q$ may be market-defined, but, for instance in cases in which no relevant market for the risk $X$ exists, it can also be interpreted as an accounting measure that is accepted by the members of the collective for the purposes of internal valuation. Since we will work in a single-period setting, there is no loss of generality in assuming that the interest rate is zero. The quantity $E^{Q}[Z]$, where the symbol $E^{Q}$ denotes expectation under the measure $Q$, then provides what will be simply referred to as the "value" (economic value, or accounting value) at time 0 of a random payoff $Z$ occurring at time 1 .

Standard financial theory dictates that the real-world measure $P$ and the valuation measure $Q$ should be equivalent in order to prevent arbitrage. There are situations, though, in which (due for instance to transaction costs or legal constraints) the participants in the collective are not free to trade the risks they share within the collective on an outside market. ${ }^{3}$ Under such circumstances, the equivalence requirement becomes less stringent. Therefore, no assumption on the relation between $P$ and $Q$ will be made in this paper; in general, the two measures are even allowed to be mutually singular. Of course, the case in which equivalence does hold is still allowed. All properties of random variables mentioned below are defined with respect to the real-world measure $P$, unless stated otherwise. In particular, expectation under the real-world measure $P$ is denoted simply by the symbol $E$.

It will be assumed throughout that the risk $X$ is nondegenerate. As mentioned above, positive values of $X$ are interpreted as gains. Appropriate sign changes can be made to cover the case in which the risk $X$ represents a liability. For convenience, it will also be assumed throughout that the risk $X$ can only take finitely many values. This assumption is not essential; however, it simplifies some of the proofs considerably. In order to interpret $E^{Q}[X]$ meaningfully as the value of the risk $X$, it is required that $X$ has finite expectation under $Q$; since the measure $Q$ in fact does not appear as such in the final problem formulation (Problem 2.9 below), this will not be stated as a separate assumption. 
The assumptions on the pooled risk $X$ can be summarized as follows for future reference.

Assumption 2.1. The risk $X$ is a finitely discrete nondegenerate random variable.

An allocation rule is a collection of functions $\left(y_{1}(\cdot), \ldots, y_{n}(\cdot)\right)$ satisfying the feasibility constraint

$$
\sum_{i=1}^{n} y_{i}(x)=x
$$

for all $x$. The meaning of these functions is that $y_{i}(x)$ is the amount received by agent $i$ when the realized outcome of the risk $X$ is $x$. As a result, the risk faced by agent $i$ after allocation is the random variable $Y_{i}:=y_{i}(X)$. An allocation rule is said to be linear if all allocation functions are of the form

$$
y_{i}(x)=a_{i} x+b_{i}
$$

where $a_{i}$ and $b_{i}$ are constants. For a linear rule, the feasibility constraint (2.1) holds if and only if the conditions

$$
\sum_{i=1}^{n} a_{i}=1, \quad \sum_{i=1}^{n} b_{i}=0,
$$

are satisfied. When all coefficients $b_{i}$ are equal to zero, we speak of a proportional allocation rule.

It will be assumed in this paper that all agents are of the von NeumannMorgenstern type, which means that their preferences can be described in terms of expected utility. In other words, the expected utility derived by agent $i$ from a risk $Y_{i}$ is given by $E\left[u_{i}\left(Y_{i}\right)\right]$, where $u_{i}(\cdot)$ is agent $i$ 's utility function. The following assumptions will be used.

Assumption 2.2. The utility functions of all agents belong to the class of realvalued functions $u(x)$ with the following properties:

$i$. The function $u(x)$ is defined on a domain of the form $(L, \infty)$, where $L$ is either finite or equal to $-\infty$.

ii. The function $u(x)$ is strictly increasing, strictly concave, and twice continuously differentiable.

iii. $\lim _{x \downarrow L} u^{\prime}(x)=\infty$.

An important function associated to the utility function is the Arrow-Pratt coefficient of risk aversion $r(x)=-u^{\prime \prime}(x) / u^{\prime}(x)$. Under the assumptions stated above, the coefficient of risk aversion is a continuous function defined on the interval $(L, \infty)$. We will use the following assumption on the behavior of the coefficient of risk aversion in the range of large losses.

Assumption 2.3. For all agents who can tolerate arbitrarily large losses (i.e., the lower bound of the domain of their utility function is $-\infty)$, the coefficient of risk 
aversion $r(x)$ satisfies

$$
\lim _{x \rightarrow-\infty} x r(x)=-\infty
$$

This assumption states that, for all agents, either the domain of the utility function is bounded below, or the condition (2.3) holds, which expresses that the coefficient of risk aversion should not tend to zero too quickly when the losses that are considered are becoming increasingly large. A rough summary of the assumption is therefore that agents should be sufficiently risk averse toward large losses. The condition (2.3) is clearly satisfied in the case of constant absolute risk aversion (exponential utility). This implies that in fact all utility functions in the hyperbolic absolute risk aversion (HARA) class are covered by Assumption 2.3, since the domains of the non-exponential utility functions in this class are bounded below.

The relevant attributes of agents in this paper consist not only of preference specifications but also of what we call claim values. The claim values of the agents will be denoted by $v_{i}$. These numbers represent ownership rights of the agents. A typical situation is that the agents hold risks $X_{i}$ which are the uncertain outcomes of what we shall refer to as the agents' projects. The risk $X$ arises as the pooled project; in other words, we have $X=\sum_{i=1}^{n} X_{i}$. The numbers $v_{i}$ are determined as the project values: $v_{i}=E^{Q}\left[X_{i}\right]$.

An allocation rule is said to be financially fair (with respect to the given pricing measure $Q$ and the claim values $v_{i}$ ) if

$$
E^{Q}\left[y_{i}(X)\right]=v_{i} \quad(i=1, \ldots, n) .
$$

In the interpretation given above, this condition becomes $E^{Q}\left[y_{i}(X)\right]=E^{Q}\left[X_{i}\right]$, which means that agents do not incur an immediate loss or gain in value by trading their private risk $X_{i}$ for a share in the pool. Of course, there may still be a change in the risk profiles of the agents, which, if the allocation rule is properly constructed, can be beneficial to all.

For linear allocation rules $y_{i}(x)=a_{i} x+b_{i}$, financial fairness means that the relation $a_{i} E^{Q}[X]+b_{i}=v_{i}$ is satisfied for all agents $i$. Due to the feasibility constraints (2.2), this can only hold if $E^{Q}[X]=\sum_{i=1}^{n} v_{i}$. Of course, this is automatically satisfied when $X=\sum_{i=1}^{n} X_{i}$ and $v_{i}=E^{Q}\left[X_{i}\right]$. Alternatively, one can take the claim values $v_{i}$ as primitive inputs, and define the number $v$ as their sum:

$$
v=\sum_{i=1}^{n} v_{i} .
$$

Then, a financially fair linear allocation rule can be defined as one of the form $y_{i}(x)=a_{i} x+b_{i}$, where the numbers $a_{i}$ and $b_{i}$ satisfy the relation $a_{i} v+b_{i}=v_{i}$ for all $i$. This means that $b_{i}=v_{i}-a_{i} v$. If $b_{i}$ is defined in this way, then the constraint $\sum_{i=1}^{n} b_{i}=0$ is satisfied when $\sum_{i=1}^{n} a_{i}=1$, and the generalform of a 
financially fair linear allocation rule becomes

$$
y_{i}(x)=v_{i}+a_{i}(x-v),
$$

where $\sum_{i=1}^{n} a_{i}=1$ and $v$ is defined by (2.5).

In this way, it becomes possible to avoid reference to the valuation measure $Q$ altogether in the problem formulation in the case of linear allocation rules. This route will be followed below (Problem 2.9). The rule (2.6) can be read as an agreement between the members of the collective to divide the payoff of the pooled project according to the values $\left(v_{1}, \ldots, v_{n}\right)$ in case the outcome of the project is equal to $v$, and to divide deviations from that outcome (positive or negative) in proportion to the numbers $a_{i}$.

The numbers $a_{i}$ are referred to as the participation coefficients. It is not imposed a priori that these coefficients should lie between 0 and 1, but from Lemma 3.4 it follows that, to achieve efficiency, they must. The problem is to choose the participation coefficients in relation to the risk appetites of the members of the collective.

Remark 2.4. Generally speaking, it is easier to establish financial fairness for linear allocation rules than to do the same for nonlinear rules. Evaluation of the lefthand side of (2.4) requires the application of a general pricing operator, which may be involved. If the pricing rule is linear, then agents only need to agree on the numbers $v_{i}$, rather than on the full pricing measure $Q$. In the case of a proportional rule $\left(b_{i}=0\right.$ for all $\left.i\right)$, the coefficients $a_{i}$ in a financially fair rule must be equal to $v_{i} / v$, and so in this case it is already sufficient if agents agree on these ratios. The relative simplicity of establishing financial fairness may be one of the reasons for the popularity of linear allocation rules and of proportional allocation rules.

Let $L_{i} \in \mathbb{R} \cup\{-\infty\}$ denote the lower bound of the domain of the utility function $u_{i}$ used by agent $i{ }^{4}$ The following assumptions will be used on the relations between the lower bounds $L_{i}$, the claim values $v_{i}$, and the minimum of the risk $X$.

Assumption 2.5. The lower bounds $L_{i}$ satisfy the inequality $\sum_{i=1}^{n} L_{i}<\min X$.

Assumption 2.6. For each agent $i \in\{1, \ldots, n\}$, the inequality $L_{i}<v_{i}$ holds.

Assumption 2.7. The sum of the claim values exceeds the minimum of the pooled risk, i.e., $v>\min X$.

Without Assumption 2.5, it is not possible to find an allocation rule $\left(y_{1}(x), \ldots, y_{n}(x)\right)$ that is admissible in the sense that for all of the agents all of the possible outcomes are in the domain of their utility function, i.e.,

$$
\min y_{i}(X)>L_{i} \quad \text { for all } i \text {. }
$$

If at least one of the lower bounds $L_{i}$ is equal to $-\infty$, then the sum of the lower bounds is taken to be equal to $-\infty$ as well, and the assumption is automatically satisfied. Assumption 2.6 ensures that $a_{i}=0$ (no participation in the pooled 
risk) is an admissible choice for each agent. Finally, Assumption 2.7 is satisfied automatically when the valuation measure $Q$ is equivalent to the statistical measure $P$, as must be the case (by absence of arbitrage) when $Q$ represents market valuation. The assumption is less compelling when $Q$ is an agreed-upon (accounting) measure, and cases in which it is not satisfied will be included below. Assumptions 2.5 and 2.6, however, will be maintained throughout the paper.

Define for each agent $i$ a positive (or infinite) number $\bar{a}_{i}$ by

$$
\bar{a}_{i}=\left\{\begin{array}{c}
\frac{v_{i}-L_{i}}{v-\min X} \quad \text { if } L_{i}>-\infty \text { and } \min X<v \\
\infty \quad \text { otherwise. }
\end{array}\right.
$$

Then, every rule with participation coefficients $a_{i} \in\left[0, \bar{a}_{i}\right)$ for all $i$ is admissible. It must be possible to satisfy the constraint $\sum_{i=1}^{n} a_{i}=1$. This is guaranteed by Assumption 2.5, as shown by the following lemma.

Lemma 2.8. Under Assumption 2.5, we have $\sum_{i=1}^{n} \bar{a}_{i}>1$, where $\bar{a}_{i}$ is defined in (2.8).

Proof. If $\min X \geq v$, then $\bar{a}_{i}=\infty$ for all $i$, so that the claim is valid. Assume now that $\min X<v$. If there is $i \in\{1, \ldots, n\}$ such that $L_{i}=-\infty$, then $\bar{a}_{i}=\infty$ and again the claim holds. Finally, assume that $L_{i}>-\infty$ for all $i$. We have

$$
\sum_{i=1}^{n}\left(v_{i}-L_{i}\right)=v-\sum_{i=1}^{n} L_{i}>v-\min X>0 .
$$

It follows that $\sum_{i=1}^{n} \bar{a}_{i}>1$ also in this case.

An allocation rule is said to be Pareto efficient if there is no allocation rule that provides a Pareto improvement, meaning that no agent is worse off, and at least one agent is better off. If we have a linear allocation rule which is such that there is no linear rule that provides a Pareto improvement, then we say that the given allocation rule is Pareto efficient within the class of all linear allocation rules, or more briefly that the rule is linearly Pareto efficient. An allocation rule is said to be linearly PEFF (linPEFF) if it satisfies both linear Pareto efficiency and financial fairness. For easy reference, we formally state the problem of finding linPEFF rules as follows.

Problem 2.9. Given a risk $X$ and $n$ agents with utility functions $u_{i}(\cdot)$ and claim rights $v_{i}$, find a vector of participation coefficients $\left(a_{1}, \ldots, a_{n}\right)$ such that the allocation rule (2.6), with $\sum_{i=1}^{n} v_{i}=v$, is Pareto efficient among all linear allocation rules.

As discussed above, since we are dealing here with linear allocations, no explicit mention of the pricing measure $Q$ is needed in the problem formulation; it is enough to have the claim values $v_{i}$ as input data. 


\section{EXISTENCE AND UNIQUENESS OF SOLUTIONS}

In order to find conditions for existence and uniqueness of solutions to Problem 2.9 , we translate the problem into a set of equations for the parameters $a_{i}$. We start (analogously to the approach of Borch, 1962) by parameterizing the linear allocation rules that are Pareto efficient among all linear allocation rules. For this purpose, introduce weights $\alpha_{i}$ that are attached to the agents, and consider the optimization problem

$$
\max _{\left(a_{i}, b_{i}\right)_{i=1, \ldots, n}} \sum_{i=1}^{n} \alpha_{i} E\left[u_{i}\left(a_{i} X+b_{i}\right)\right] \quad \text { subject to } \sum_{i=1}^{n} a_{i}=1, \quad \sum_{i=1}^{n} b_{i}=0 .
$$

Since we have assumed that the utility functions are strictly concave and strictly increasing, and since the constraint set is convex, it follows (see for instance Barrieu and Scandolo, 2008) that a linear allocation rule is Pareto efficient among all linear allocation rules if and only if, for some weight vector $\left(\alpha_{1}, \ldots, \alpha_{n}\right)$ with $\alpha_{i}>0$ for all $i$, it is a solution to the optimization problem (3.1). From the assumed strict concavity of the utility functions, it follows that the solution to the weighted optimization problem is unique and can be found from the first-order conditions. The Lagrangian function is

$$
\sum_{i=1}^{n} \alpha_{i} E\left[u_{i}\left(a_{i} X+b_{i}\right)\right]-\lambda\left(\sum_{i=1}^{n} a_{i}-1\right)-\mu \sum_{i=1}^{n} b_{i},
$$

where $\lambda$ and $\mu$ are Lagrange multipliers. Therefore, the first-order conditions can be written as

$$
\begin{aligned}
\alpha_{i} E\left[X u_{i}^{\prime}\left(a_{i} X+b_{i}\right)\right] & =\lambda & & (i=1, \ldots, n), \\
\alpha_{i} E\left[u_{i}^{\prime}\left(a_{i} X+b_{i}\right)\right] & =\mu & & (i=1, \ldots, n) .
\end{aligned}
$$

In other words, the pair of parameter vectors $\left(a_{1}, \ldots, a_{n}\right)$ and $\left(b_{1}, \ldots, b_{n}\right)$ satisfying (2.2) represents a linearly Pareto efficient solution if and only if there exist $\alpha_{1}, \ldots, \alpha_{n}$, with $\alpha_{i}>0$ for all $i$, and real numbers $\lambda, \mu$ such that the conditions (3.2)-(3.3) are satisfied. This leads to the following conclusion (see the appendix for a proof).

Theorem 3.1. A linear allocation rule given by the parameter vectors $\left(a_{1}, \ldots, a_{n}\right)$ and $\left(b_{1}, \ldots, b_{n}\right)$ is Pareto efficient among all linear allocation rules if and only if there is a constant $c$, not depending on the agent index $i$, such that

$$
\frac{E\left[X u_{i}^{\prime}\left(a_{i} X+b_{i}\right)\right]}{E\left[u_{i}^{\prime}\left(a_{i} X+b_{i}\right)\right]}=c,
$$

for all $i=1, \ldots, n$.

Condition (3.4) is notably different from Borch's condition for Pareto efficiency of general allocation rules. The condition given by Borch (1962) is that 
an allocation rule $\left(y_{1}(x), \ldots, y_{n}(x)\right)$ is Pareto efficient if and only if there exists a function $c(x)$, not depending on the agent index $i$, and positive constants $\alpha_{i}$ such that

$$
\alpha_{i} u_{i}^{\prime}\left(y_{i}(x)\right)=c(x),
$$

for all $x$ in the support of $X$ and for all $i=1, \ldots, n$. One of the differences is that the condition (3.4) depends on the probabilities of the outcomes of $X$, whereas Borch's condition does not. A heuristic discussion of the relation between the "linear" condition (3.4) and the "nonlinear" condition (3.5) is given in Section A in the appendix.

Remark 3.2. Since Pareto efficiency among linear allocation rules is a weaker condition than Pareto efficiency among all allocation rules, the condition (3.4) should be a consequence of (3.5) in case $y_{i}(x)=a_{i} x+b_{i}$. To see this explicitly, define for each $i$, and for a given set of allocation functions $y(x)=$ $\left(y_{1}(x), \ldots, y_{n}(x)\right)$, a function $f_{i}^{y}(x)$ by

$$
f_{i}^{y}(x)=\frac{u_{i}^{\prime}\left(y_{i}(x)\right)}{E\left[u_{i}^{\prime}\left(y_{i}(X)\right)\right]} .
$$

For each $i$, the random variable $f_{i}^{y}(X)$ is positive and satisfies $E\left[f_{i}^{y}(X)\right]=1$; therefore, it defines a change of measure from the measure $P$ to a new measure $P_{i}^{y}$ (see the remark below for an interpretation of $P_{i}^{y}$ ). If (3.5) holds, then, for all $i=1, \ldots, n$,

$$
f_{i}^{y}(x)=\frac{u_{i}^{\prime}\left(y_{i}(x)\right)}{E\left[u_{i}^{\prime}\left(y_{i}(X)\right)\right]}=\frac{c(x)}{E\left[\alpha_{i} u_{i}^{\prime}\left(y_{i}(X)\right)\right]}=\frac{c(x)}{E[c(X)]},
$$

so that in particular $f_{i}^{y}(x)=f_{j}^{y}(x)$ for all $i$ and $j$. In other words, $P_{i}^{y}=P_{j}^{y}$ for all $i$ and $j$. Conversely, if $P_{i}^{y}=P_{j}^{y}$ for all $i$ and $j$, then it follows that $f_{i}^{y}(x)=f_{j}^{y}(x)=$ (say) $f^{y}(x)$ for all $x$ in the support of $X$ and for all $i$ and $j$, so that Borch's condition is satisfied with $c(x)=f^{y}(x)$ and $\alpha_{i}=1 / E\left[u_{i}^{\prime}\left(y_{i}(X)\right)\right]$. Borch's condition is therefore equivalent to the condition that the measures $P_{i}^{y}$ are the same for all $i$. Another equivalent formulation is that $E_{i}^{y}[Z]=E_{j}^{y}[Z]$ for all $i, j=1, \ldots, n$ and for all $\sigma(X)$-measurable random variables $Z$, where $E_{i}^{y}$ denotes expectation under $P_{i}^{y}$. This last formulation allows direct comparison with the condition for linear Pareto efficiency (3.4), which, using $y_{i}(x)=a_{i} x+b_{i}$, can be rewritten as the condition that $E_{i}^{y}[X]=E_{j}^{y}[X]$ for all $i, j=1, \ldots, n$.

Remark 3.3. The random variable $f_{i}^{y}(X)$ can be interpreted in terms of indifference pricing. Agent $i$ 's marginal indifference price $\pi_{i}(Z)$ (see for instance Carmona (2009)) for a contingent payoff $Z$ to be obtained from the risk pool, given the allocation $y_{i}(X)$, is the price at which agent $i$ is neither inclined to buy nor inclined to sell a small quantity of the payoff $Z$, i.e.,

$$
\left.\frac{d}{d \varepsilon} E\left[u_{i}\left(y_{i}(X)+\varepsilon\left(Z-\pi_{i}(Z)\right)\right)\right]\right|_{\varepsilon=0}=0 .
$$


From this, one finds that $\pi_{i}(Z)=E\left[f_{i}^{y}(X) Z\right]=E_{i}^{y}[Z]$. Consequently, the random variable $f_{i}^{y}(X)$ can be described as the indifference pricing kernel for agent $i$ associated to the allocation rule $\left(y_{1}(x), \ldots, y_{n}(x)\right)$. Borch's efficiency condition is that indifference prices of all contingent claims on the risk pool are the same for all agents. In contrast, the condition (3.4) requires only that the indifference price of total risk $X$ should be the same for all agents. This is natural because only the total risk is traded in a linear risk exchange, aside from cash which is always priced the same.

We have not assumed a priori that the participation coefficients $a_{i}$ in a linear allocation rule should be positive. By the characterization in Theorem 3.1, we can prove that positivity of these coefficients must hold in all Pareto efficient rules, in fact even in all linearly Pareto efficient rules. Since the sum of the $a_{i}$ 's must be equal to 1 , this also shows that the participation coefficients in an efficient rule must lie between 0 and 1 .

Lemma 3.4. If a linear allocation rule given by parameter vectors $\left(a_{1}, \ldots, a_{n}\right)$ and $\left(b_{1}, \ldots, b_{n}\right)$ is linearly Pareto efficient, then the parameters $a_{i}$ are all positive.

Proof. Suppose that the linear allocation rule given by $\left(a_{1}, \ldots, a_{n}\right)$ and $\left(b_{1}, \ldots, b_{n}\right)$ is Pareto efficient among all linear allocation rules. The rule must then satisfy the condition (3.4), from which it follows that

$$
\operatorname{Cov}\left(X, u_{i}^{\prime}\left(a_{i} X+b_{i}\right)\right)=(c-E[X]) E\left[u_{i}^{\prime}\left(a_{i} X+b_{i}\right)\right],
$$

for all $i$. The sign of the right-hand side in this expression is the same for all $i$, since $E\left[u_{i}^{\prime}\left(a_{i} X+b_{i}\right)\right]$ is always positive, and the factor $c-E[X]$ does not depend on $i$. The sign of the left-hand side is positive if $a_{i}$ is negative and vice versa, since the random variables $X$ and $u_{i}^{\prime}\left(a_{i} X+b_{i}\right)$ are comonotonic in the first case and countermonotonic in the latter case. ${ }^{5}$ The left-hand side is zero if $a_{i}$ is zero. From the feasibility constraint $\sum_{i=1}^{n} a_{i}=1$, it follows that at least one of the coefficients $a_{i}$ must be positive. Consequently, the relation (3.7) implies that all of those coefficients must be positive.

Remark 3.5. From the reasoning used in the proof, it also follows that the constant c appearing in (3.4) must be less than $E[X]$.

Among the Pareto efficient solutions as determined by Theorem 3.1, we now want to look for allocation rules that satisfy the financial fairness condition (2.4), and hence are of the form (2.6). We arrive at the following conclusion.

Theorem 3.6. A vector $\left(a_{1}, \ldots, a_{n}\right)$ provides a solution to Problem 2.9 if and only if

$$
\sum_{i=1}^{n} a_{i}=1,
$$

and, for some constant $c$, 


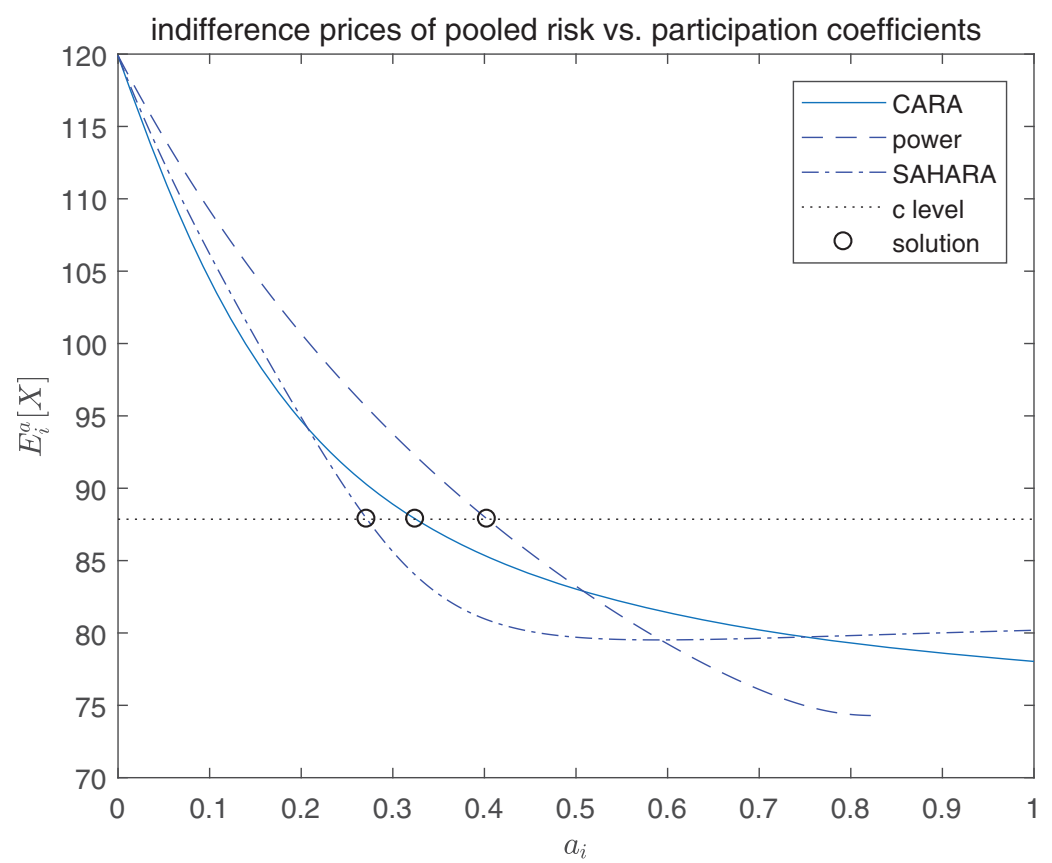

FIGURE 1: Example of computation of participation coefficients. The level $c$ is adjusted to ensure that the numbers on the horizontal axis corresponding to the locations of intersections with the indifference price curves add up to 1 . Parameter values are as follows (referring to Table 1 for utility parameters):

$X \sim 100 \cdot \operatorname{Lognormal}(0.25,0.2)$ (discretized on range $[74.3,181.7]), v_{1}=40, v_{2}=30, v_{3}=40$, CARA: $\alpha=0.2$, power: $\gamma=4, b=0$, SAHARA: $\gamma=2, \beta=5, d=30$. Solution: $a_{1}=32.5 \%, a_{2}=40.3 \%, a_{3}=27.2 \%$.

$$
\frac{E\left[X u_{i}^{\prime}\left(a_{i}(X-v)+v_{i}\right)\right]}{E\left[u_{i}^{\prime}\left(a_{i}(X-v)+v_{i}\right)\right]}=c,
$$

for all $i$.

A possible approach to solving this set of equations is the following. For a given initial guess of the constant $c$, find corresponding values $a_{i}(i=1, \ldots, n)$ such that (3.9) holds. Verify whether these values satisfy condition (3.8). If not, adapt the constant $c$ and try again. This idea is illustrated in Figure $1 .{ }^{6}$ In comparison, the procedure to compute the general (nonlinear) PEFF solution is considerably more complicated, as can be expected since there are many more unknowns in the general PEFF case than in the linPEFF case. The problem to be solved in the general case can be formulated as follows: determine positive constants $\alpha_{i}(i=1, \ldots, n)$ and continuous functions $c(x)$ and $y_{i}(x)(i=1, \ldots, n)$ such that, for all $x$ and all $i=1, \ldots, n$,

$$
\sum_{i=1}^{n} y_{i}(x)=x, \quad \alpha_{i} u_{i}^{\prime}\left(y_{i}(x)\right)=c(x), \quad E^{Q}\left[y_{i}(X)\right]=v_{i} .
$$


Sufficient conditions for unique solvability of this set of equations were obtained by Gale (1977), Gale and Sobel (1979), and Bühlmann and Jewell (1979). An iterative computational procedure for the computation of solutions has been proposed by Pazdera et al. (2017).

Sufficient conditions for existence and uniqueness of solutions to the linPEFF equations (3.8)-(3.9) will now be discussed. In order to simplify the notation, write

$$
E_{i}^{a_{i}}[X]:=\frac{E\left[X u_{i}^{\prime}\left(a_{i}(X-v)+v_{i}\right)\right]}{E\left[u_{i}^{\prime}\left(a_{i}(X-v)+v_{i}\right)\right]} .
$$

The functions $a_{i} \mapsto E_{i}^{a_{i}}[X]$ are defined on the intervals $\left[0, \bar{a}_{i}\right)$, where the constants $\bar{a}_{i}$ are defined by (2.8). In the theorem below, a function defined on an interval $[a, b]$ is said to be "piecewise monotonic" if there exists a partitioning of the interval of definition into finitely many intervals such that the function is monotonic on each of these subintervals. An example of a continuously differentiable function that is not piecewise monotonic is the one defined by $f(0)=0$ and $f(x)=x^{3} \sin (1 / x)$ for $x \neq 0$ on the interval $[-1,1]$. The assumption of piecewise monotonicity does not seem unduly restrictive for actuarial applications. The proof of the theorem is given in the appendix.

Theorem 3.7. Under assumptions 2.1, 2.2, 2.3, 2.5, 2.6, and 2.7, Problem 2.9 admits a solution if all functions $a_{i} \mapsto E_{i}^{a_{i}}[X]$ are piecewise monotonic. If all functions $a_{i} \mapsto E_{i}^{a_{i}}[X]$ are strictly monotonic, then the solution is determined uniquely.

A sufficient condition for unique solvability that is stated more directly in terms of the utility functions can be given as follows. The proof can be found in the appendix.

Corollary 3.8. Under assumptions 2.1, 2.2, 2.3, 2.5, 2.6, and 2.7, Problem 2.9 admits a unique solution if the functions $x \mapsto(x-v) r_{i}\left(a_{i}(x-v)+v_{i}\right)$ defined on $[\mathrm{min} X, \infty)$ are strictly increasing in $x$ for all $i$ and for all fixed values of $a_{i} \in$ $\left[0, \bar{a}_{i}\right)$.

The condition in the corollary is clearly satisfied if all agents have constant absolute risk aversion. Another situation of particular interest is the one in which agents' preferences are described by shifted power utility (see Table 2). The coefficients of absolute risk aversion are in this case given by functions of the form

$$
r_{i}(y)=\frac{\gamma_{i}}{y-L_{i}},
$$

with $\gamma_{i}>0$, so that

$$
(x-v) r_{i}\left(a_{i}(x-v)+v_{i}\right)=\frac{\gamma_{i}(x-v)}{a_{i}(x-v)+v_{i}-L_{i}} .
$$

The denominator of the expression on the right-hand side is positive by construction for $x \geq \min X$ and $0 \leq a_{i}<\bar{a}_{i}$. As $x$ increases from min $X$, the 
numerator is first negative and then positive. We can conclude that the expression above describes part of an increasing branch of a hyperbola. In particular, it follows that the sufficient condition of the corollary is satisfied for collectives of shifted power utility agents.

Remark 3.9. As noted above, one may imagine situations in which Assumption 2.7 is not satisfied. In case the sum of the claim values is exactly equal to the minimal value of the risk $X$, one can verify that a sufficient condition for existence of a solution to Problem 2.9 is that $\lim _{x \rightarrow \infty} u_{i}^{\prime}(x)=0$ for all agents $i$. This is, of course, a quite common condition for utility functions. The asymptotic condition of Assumption 2.3 is not needed in this case. When the sum of the claim values is less than the minimum of the risk $X$, the asymptotic condition of Assumption 2.3 should be replaced by an asymptotic condition that applies to large gains rather than to large losses, namely

$$
\lim _{x \rightarrow \infty} \operatorname{xr}(x)=\infty
$$

It can be shown that satisfaction of this property by the utility functions of all agents, in combination with assumptions 2.1, 2.2, 2.5, 2.6, and the assumption $\min X>v$, is sufficient for the existence of a solution to Problem 2.9. The proof is analogous to the proof of Theorem 3.7 that is given in the appendix. Condition (3.11) may be compared to the condition of "reasonable asymptotic elasticity", which has been introduced by Kramkov and Schachermayer (1999), and which, under regularity conditions, can be formulated as the condition $\lim _{x \rightarrow \infty} x r(x)>0$ (Biagini and Guasoni, 2011). Clearly, (3.11) is a strengthening of the reasonable asymptotic elasticity condition.

\section{PARTICULAR CASES}

While there are typically no efficient linear allocation rules unless agents are equicautious, the deviation of linear rules from efficiency may be small. In practice, then, the simpler linear allocation rule may still be preferred. In this section, we consider financially fair risk sharing between agents of different types in a few specific cases, in order to gain insights into the circumstances under which the benefits from nonlinear risk sharing could be substantial.

We use agents of four different types, namely constant absolute risk aversion, power utility, SAHARA utility, and kinked utility. The marginal utilities and risk aversion functions corresponding to these types are listed in Table 1. The SAHARA type and the kinked type are taken from Chen et al. (2011) and Dai and Schumacher (2009), respectively. An offset is introduced, since we interpret the argument of the utility function as the revenues received by a given agent as a result of the joint project with outcome $X$; in general, we can expect that agents do have other sources of wealth in addition to the revenues from the project. The offset appears explicitly in power utility and in kinked utility as a parameter $b$ 
TABLE 1

UTILITY SPECIFICATIONS.

\begin{tabular}{lccc}
\hline \hline Type & Marginal Utility & Risk Aversion & Constraints \\
\hline CARA & $\exp (-\alpha x)$ & $\alpha$ & $\alpha>0$ \\
Power & $(x+b)^{-\gamma}$ & $\gamma /(x+b)$ & $\gamma>0, x>-b$ \\
SAHARA & $\left(x-d+\sqrt{\beta^{2}+(x-d)^{2}}\right)^{-\gamma}$ & $\gamma /\left(\sqrt{\beta^{2}+(x-d)^{2}}\right)$ & $\gamma>0$ \\
& $e^{\eta}(x+b)^{-\gamma}\left(x \leq d_{1}\right)$ & & \\
Kinked & $(x+b)^{-\gamma}\left(d_{1}<x<d_{2}\right)$ & $\frac{\gamma}{x+b}+\eta\left(\delta_{d_{1}}+\delta_{d_{2}}\right)$ & $\gamma>0, \eta \geq 0$ \\
& $e^{-\eta}(x+b)^{-\gamma}\left(x \geq d_{2}\right)$ & & $x>-b$
\end{tabular}

See Wuerth and Schumacher (2011) on how to represent risk aversion in the kinked case. Symbol $\delta_{z}$ denotes the Dirac delta function located at $z$.

(basis wealth); in the case of SAHARA utility, it is included in the location parameter $d$. Kinked utility differs from power utility by jumps in risk aversion at two levels, which are thought of as "minimally satisfactory" and "satisfactory", respectively. The specification of kinked utility does not satisfy the smoothness conditions that have been imposed in the theorems above; the nonsmoothness does not lead to numerical problems, however, and we conjecture that all results can be extended.

In the first three examples, we work with two agents who are both owners of (a discretized version of) a lognormal risk. The risks correspond to investments in a Black-Scholes world on a five-year horizon. We allow for varying degrees of correlation between the two risks.

The benefit of risk sharing will be quantified in terms of the agents' reservation prices for taking part in the proposed collective. This is the maximum amount that a given agent would rationally be willing to pay for participation in the pool in the absence of alternative forms of risk sharing. Let the risk held by agent $i$ in autarky be denoted by $X_{i}$. Then, the reservation price is computed as the number $c_{i}$ such that

$$
E\left[u_{i}\left(X_{i}\right)\right]=E\left[u_{i}\left(Y_{i}-c_{i}\right)\right],
$$

where $Y_{i}$ represents the agent's claim risk that ensues from the proposed risk sharing agreement. ${ }^{7}$ In general, there is no a priori guarantee that the number $c_{i}$ is positive. It may happen that the constraint of financial fairness, in combination with the characteristics of agents participating in a proposed pool, works in such a way that the risk sharing agreement is not beneficial for one or more of the participants. In this case, if we interpret the allocation rule as a proposal that is made to a group of individuals acting rationally, then the proposal would fail. Changing from a linear allocation rule to a more general nonlinear rule may then bring better results. If the participants are willing to accept deviations from 
financial fairness to a certain extent, then of course even wider possibilities open up. Generally speaking, whether it is a good idea for a given group of individuals or companies to form a risk sharing collective depends on many factors, including the degree of dependence between their risk exposures and the spread in their attitudes toward risk. It is beyond the scope of this paper to address this issue formally, but the examples below may provide some indications. We do find that $c_{i}$ is positive for all agents at least in some cases.

It should be noted that reservation prices use evaluation of utility at a claim of the form $Y_{i}-c$, whereas we have formulated Pareto efficiency in terms of expected utility arising from the claim $Y_{i}$ itself. The evaluation by reservation prices is therefore not quite the same as the evaluation used in the definition of efficiency. As a result, reservation prices associated to a solution that is not Pareto efficient may be higher for all agents than reservation prices for an efficient solution, in particular when the proposed inefficient solution is actually close to efficiency.

In the examples, the projects owned by the agents produce an approximately lognormally distributed outcome and have economic value $v_{i}$ equal to 50 . The complete specifications are given in Table 2. The risk-neutral measure $Q$ is constructed in such a way that the risks are also approximately lognormal under $Q$ with the same correlation. The interest rate is taken to be zero for simplicity; in other words, the expectations of the outcomes of the two autarky projects under $Q$ are both equal to 50 . The pooled risk $X$ is formed simply as the sum of the two autarky projects; this means that some possible benefits of cooperation, such as accessibility of a wider range of investment opportunities, are ignored. Three possible forms of risk sharing agreements are considered:

- the proportional scheme, which divides the outcome of $X$ proportionally among the participants on the basis of the ratio of the agent's claim value to the value of the total risk;

- the linPEFF rule;

- the general PEFF rule (i.e., not restricted by linearity).

The proportional scheme is determined completely by the financial fairness constraint. Each agent receives the percentage of the total claim that is equal to the value of the agents' autarky project relative to the total value of the pooled projects. In the first series of examples, the proportional rule therefore is simply that each agent receives one-half of the outcome of the pooled risk.

We consider situations in which the risks are uncorrelated, partially correlated, and fully correlated. When the correlation is less than complete, agents enjoy a diversification benefit from pooling. Even in the case of full correlation, however, agents may still benefit from forming a collective when they have different attitudes toward risk. In this case, the beneficial effect comes purely from the redistribution of risk, which is possible even under the constraints of financial fairness and a linear sharing rule.

In our first example, we consider risk sharing between a CARA agent and a power utility agent. The risk aversion coefficient of the CARA agent is 0.04 . For 


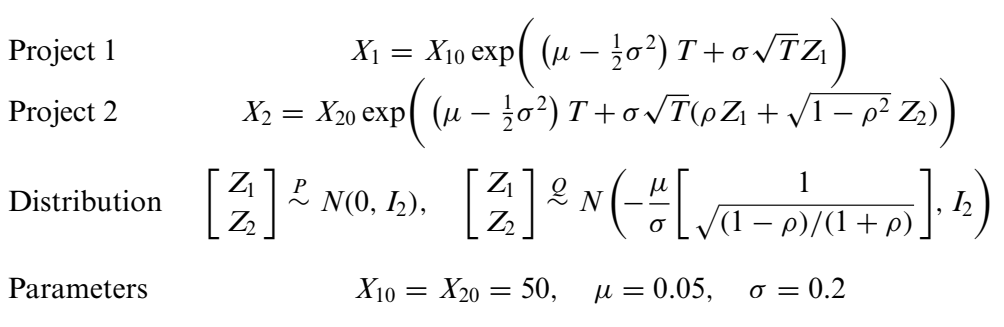

The correlation $\rho$ may take several values, as discussed in the specific examples. Symbol $I_{2}$ denotes the $2 \times 2$ unit matrix. Actual distributions used are discrete approximations of the indicated lognormal distributions.

TABLE 3

RESERVATION PRICES FOR RISK SHARING BETWEEN A CARA AGENT AND A POWER UTILITY AGENT.

\begin{tabular}{lcccccccc}
\hline \hline & \multicolumn{2}{c}{0} & & \multicolumn{2}{c}{0.5} & & \multicolumn{2}{c}{1} \\
\cline { 2 - 3 } \cline { 8 - 9 } Correlation & CARA & Power & & CARA & Power & & CARA & Power \\
\hline Proportional & 4.83 & 7.00 & & 2.19 & 2.98 & & 0 \\
linPEFF & 4.79 & 9.37 & & 0.54 & 7.34 & & -2.98 & 5.82 \\
Nonlinear PEFF & 4.93 & 9.28 & & 0.88 & 7.06 & & -2.39 & 5.30 \\
linPEFF Coefficient & $67 \%$ & $33 \%$ & & $68 \%$ & $32 \%$ & & $69 \%$ & $31 \%$ \\
\hline \hline
\end{tabular}

Parameters: $\alpha=0.04$ (CARA agent), $\gamma=5, b=10$ (power utility agent). Autarky projects as specified in Table 2. The final row shows the coefficients $a_{i}$ in the linear risk sharing rule (2.6).

the power utility agent, we take the risk aversion parameter $\gamma$ equal to 5 , and we set the offset (basis wealth) equal to 10 . Relative to the project value 50, this offset is fairly small; such a relationship might occur when the agent's project represents pension savings. The absolute risk aversion of agent 2 is decreasing; at the $5 \%$ quantile of the distribution of the agent's risk under autarky, the coefficient value is a bit more than 0.13 , whereas at the $95 \%$ quantile, it is a bit less than 0.04. Broadly speaking, this agent is therefore more risk averse than the CARA agent. The reservation prices are shown in Table 3 for different values of the correlation coefficient. ${ }^{8}$ The table also shows the risk sharing coefficients corresponding to the linPEFF solution in percentages of the deviation of the total risk from its benchmark value.

In the case of full correlation, the proportional scheme comes down to the same as autarky, and therefore the corresponding reservation prices are zero. Both the linear and the general PEFF scheme will reallocate the risk toward the CARA agent, as may be expected from the fact that this agent is less risk averse. Due to the absence of a diversification effect, the CARA agent does not benefit from the transfer of risk; rationally speaking, the pool can therefore not 
TABLE 4

RESERVATION PRICES FOR RISK SHARING BETWEEN A CARA AGENT AND A SAHARA AGENT.

\begin{tabular}{|c|c|c|c|c|c|c|}
\hline \multirow[b]{2}{*}{ Correlation } & \multicolumn{2}{|r|}{0} & \multicolumn{2}{|c|}{0.5} & \multicolumn{2}{|c|}{1} \\
\hline & CARA & SAHARA & CARA & SAHARA & CARA & SAHARA \\
\hline Proportional & 7.00 & 7.14 & 3.00 & 3.07 & 0 & 0 \\
\hline $\operatorname{linPEFF}$ & 5.69 & 8.22 & 2.48 & 3.56 & 0.51 & -0.51 \\
\hline Nonlinear PEFF & 3.97 & 9.79 & -0.67 & 6.76 & -3.74 & 3.96 \\
\hline linPEFF Coefficient & $57 \%$ & $43 \%$ & $52 \%$ & $48 \%$ & $49 \%$ & $51 \%$ \\
\hline
\end{tabular}

Parameters: $\alpha=0.1$ (CARA agent), $\gamma=2, \beta=5, d=60$ (SAHARA agent). Autarky projects as specified in Table 2. The final row shows the coefficients $a_{i}$ in the linear risk sharing rule (2.6).

be formed. When correlation is higher, pooling does become attractive also for the CARA agent. In the uncorrelated case, both agents profit substantially, but it is still the less risk averse agent who benefits most. There is not much difference between results from linear or nonlinear risk sharing.

In the second example, we consider risk sharing between a CARA agent and a SAHARA agent. The CARA agent has risk aversion coefficient 0.1 . Parameters for the SAHARA agent are chosen in such a way that the risk aversion of the SAHARA agent is higher than 0.1 in the vicinity of the critical level $d=60$, but is otherwise less than that. Overall, the agents can be described as approximately equally risk averse. Results are shown in Table 4 . The agents benefit in about the same way from proportional risk sharing, as long as there is less than full correlation. Application of the linPEFF rule is not advantageous for the SAHARA agent in the case of full correlation, but is beneficial to both when the correlation is 0.5 or zero. The results of the nonlinear PEFF rule are quite different; there are strong advantages to this rule for the SAHARA agent at all levels of correlation, but the CARA agent will not be willing to participate on these terms unless the correlation is quite low.

For the third example, we introduce an agent with kinked utility, and we consider risk sharing between this agent and a power utility agent. The latter agent in fact has the same utility function as the first, but without the kinks. Out of these two, the kinked utility agent is therefore to be considered as the one who is globally more risk averse. Unlike the situation in the first two examples, both agents profit from risk sharing even in the case of full correlation. In this case, the benefits from risk sharing are purely due to the reshaping of the distributions of the risks confronting the agents, rather than to diversification. The positive effects of diversification are clearly noticeable in the situations of incomplete correlation. From the results shown in the table, not much difference is to be seen between the linear rule and the nonlinear rule. Actually, in the case of zero correlation, both agents associate a higher reservation price to the linear rule than to the nonlinear rule. As noted before, this may happen because evaluation in terms of reservation prices does not match entirely with evaluation in terms 
TABLE 5

RESERVATION PRICES FOR RISK SHARING BETWEEN A POWER UTILITY AGENT AND A KINKED UTILITY AGENT.

\begin{tabular}{lcccccccc}
\hline \hline \multirow{2}{*}{ Correlation } & \multicolumn{2}{c}{0} & & \multicolumn{2}{c}{0.5} & & \multicolumn{2}{c}{1} \\
\cline { 2 - 3 } \cline { 8 - 9 } & Power & Kinked & & Power & Kinked & & Power & Kinked \\
\hline Proportional & 2.85 & 4.54 & & 1.34 & 2.03 & & 0 & 0 \\
linPEFF & 5.14 & 3.42 & & 2.50 & 2.20 & & 0.24 & 1.16 \\
Nonlinear PEFF & 5.08 & 3.36 & & 2.44 & 2.23 & & 0.21 & 1.26 \\
linPEFF Coefficient & $70 \%$ & $30 \%$ & & $69 \%$ & $31 \%$ & & $67 \%$ & $33 \%$ \\
\hline \hline
\end{tabular}

Parameters: $\gamma=2, b=50$ (power utility agent), $\gamma=2, b=50, \eta=0.5, d_{1}=40, d_{2}=60$ (kinked utility agent). Autarky projects as specified in Table 2. The final row shows the coefficients $a_{i}$ in the linear risk sharing rule (2.6).

of utility. Direct calculation confirms that the utility level of the kinked utility agent is in fact higher under the nonlinear rule than under the linear rule.

The results may be illustrated graphically. Figure 2 shows the allocations, in each of the three examples, according to the linPEFF rule and according to the general PEFF rule, all in the situation in which the value of the correlation coefficient between the autarky projects is 0.5 . The values of the pooled risk $X$ on the horizontal axis cover the distribution of $X$ from the $0.5 \%$ quantile to the 99.5\% quantile. In the first example, it is seen that the linPEFF rule and the nonlinear PEFF rule are almost identical across a wide range of outcomes. The allocation function of the CARA agent is generally steeper, because this agent is less risk averse in the example than the power utility agent. Only in the case of very high outcomes, the nonlinear rules deviates and divides the outcomes more equally between the agents than the linear rule would do. In the second example, the risk aversion of the SAHARA agent is high in the vicinity of the outcome 60 , which roughly corresponds to the outcome 120 for the total risk. As a consequence, the allocation function of the SAHARA agent is more flat in this region, which necessarily means that the curve of the other agent is steeper there. Both in the regions below and above, the situation is reversed. The plot for the third example shows that the allocations according to the linear and the nonlinear allocation rules are broadly in line with each other, which explains that the reservation prices for these rules are similar. However, the nonlinear rule is able to keep the outcome for the kinked utility agent exactly at the critical levels 40 and 60 across certain ranges of the outcomes of total risk. By its nature, the linear rule cannot duplicate this behavior.

The linear rule and the nonlinear rule can also be compared by means of what might be called the profit/loss share of agents. Given an allocation function $y_{i}(x)$, the profit/loss share is defined by

$$
a_{i}(x)=\frac{y_{i}(x)-y_{i}(v)}{x-v} .
$$



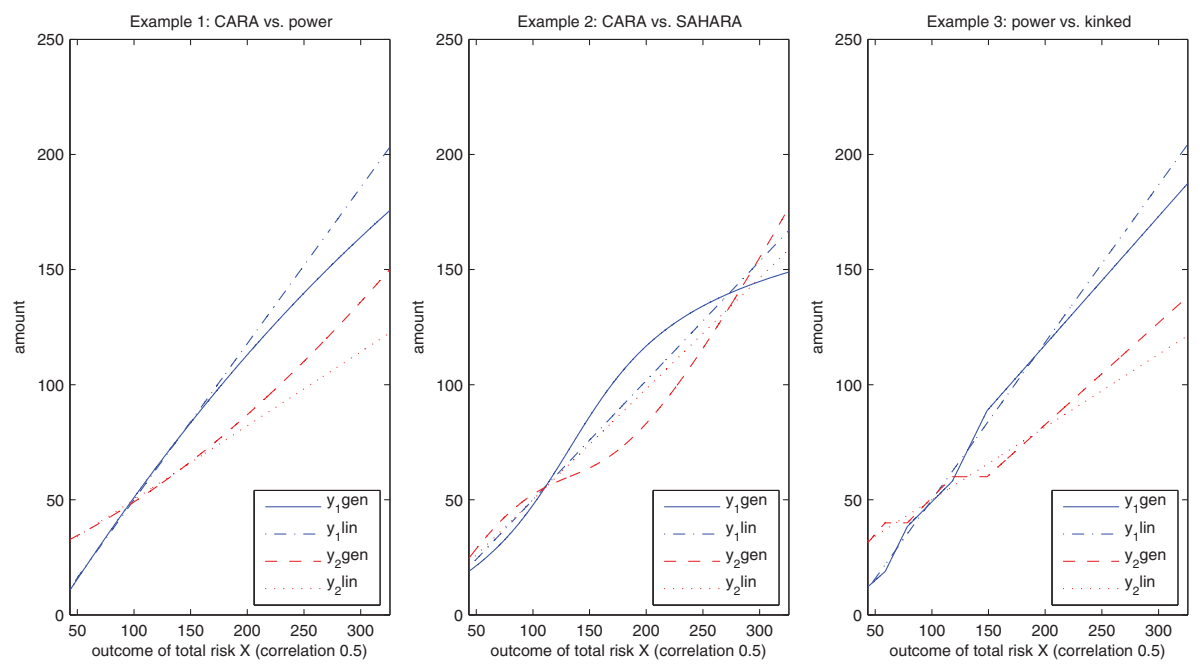

FIGURE 2: Monetary allocations in the three examples.

With this definition, the allocation rule can be written as

$$
y_{i}(x)=a_{i}(x)(x-v)+v_{i}+c_{i},
$$

where $c_{i}:=y_{i}(v)-v_{i}$ is called the "compensation". Written in this form, the nonlinear rule can be compared more easily to the linear rule (2.6). Specifically, in the linear rule the profit/loss share is constant and the compensation is zero. In the nonlinear rule, the percentage share of each agent in the loss or gain of the realized outcome with respect to the benchmark value $v$ depends on how large the deviation is. For instance, the plot corresponding to the first example in Figure 3 shows a decreasing profit/loss share for the CARA agent, which means that this agent takes a larger percentage of gains than of losses. The agent is compensated for that first by the fact that, for small gains, the percentage allocated is still higher than would follow from the linear rule, and second by the compensation amount that appears in (4.3). In the second example, the profit/loss share shows a nonmonotonic behavior. The same is true, and even more so, in the third example. In the latter two examples, the profit/loss share varies approximately between $50 \%$ and $80 \%$, which may be viewed as a substantial difference between the linear and the nonlinear rule.

For a final set of examples, we now look at a situation with three agents and focus in particular on the impacts of the basis wealth (see footnote 4) and the capital brought in by each of the agents. The agents' preferences are supposed to be given by shifted power utility, and their autarky projects are as in the previous examples. It will be assumed that the driving risk for these projects is the same as the one for the pooled project; this represents a situation in which the benefits of cooperation arise only from risk redistribution, without additional support 

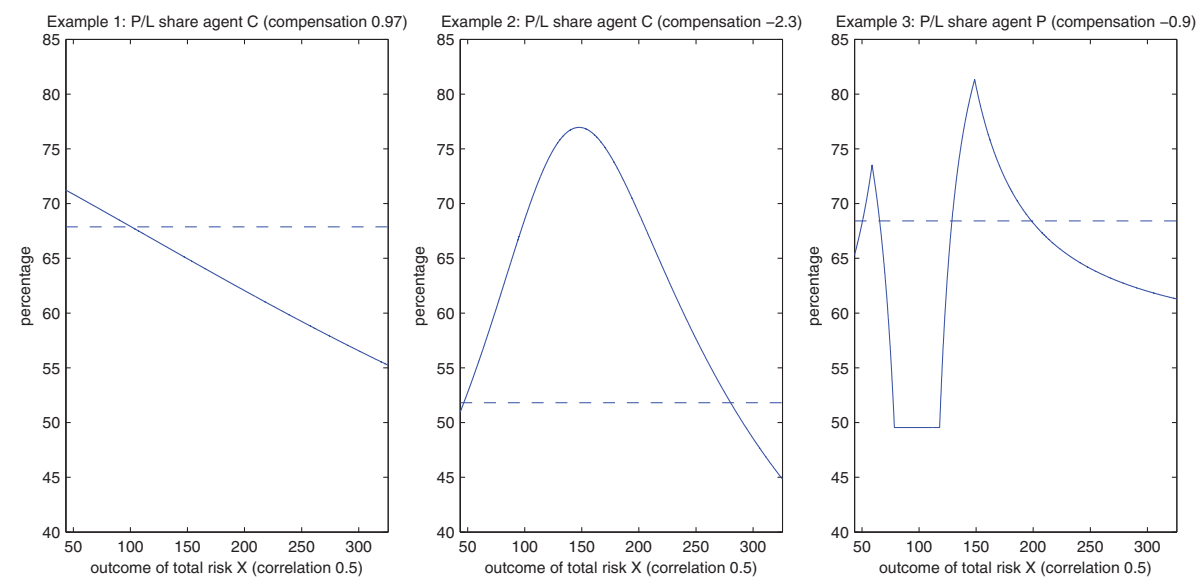

FIGURE 3: Profit/loss shares in the three examples. The dashed line corresponds to the linPEFF rule.

from diversification. The evaluation measure $Q$ relates to the statistical measure $P$ in the same way as before.

The results for four different cases are shown in Table 6. As a benchmark, first consider a situation in which all agents are the same except for their coefficients of risk aversion. Unsurprisingly, the linPEFF redistribution calls for the least risk averse agent to carry most of the risk. This agent, however, would not participate voluntarily, in contrast to the other two agents. If it is assumed that the basis wealth differs among the three agents in the sense that the least risk averse agent has the largest basis wealth (top right panel in Table 6), then the difference in risk tolerance becomes larger and, consequently, even more risk is shifted to the least risk averse agent. The least risk averse agent, whose pockets have become deeper compared to the first case, now finds the agreement attractive relative to autarky. The most risk averse agent hardly participates in the risk anymore after redistribution, and a very substantial benefit for this agent results.

The bottom left panel shows a situation where basis wealth is the same for all agents, but the agents bring in different amounts of capital, with the largest amount being supplied by the least risk averse agent. Relative to the amount of capital brought in, the benefit of the most risk averse agent is still the largest, while participation of the least risk averse agent depends on the possibility of coercion. Again, a larger basis wealth for the least risk averse agent makes the linPEFF solution acceptable to all, as shown in the bottom right panel.

The difference between the linear solution and the general (nonlinear) solution is fairly small in all four cases, and graphs are not shown. Compared to the linPEFF solution, the general PEFF solution typically allocates a somewhat larger amount to the most risk tolerant agent for high outcomes as well as for low outcomes, whereas the other two agents are slightly better off from the PEFF solution than from the linPEFF solution in the case of intermediate outcomes. 
TABLE 6

PEFF RISK SHARING OF AN APPROXIMATELY LOGNORMAL RISK BETWEEN THREE POWER UTILITY AGENTS.

\begin{tabular}{|cccc|}
\hline Agent & 1 & 2 & 3 \\
\hline$\gamma_{i}$ & 2 & 5 & 10 \\
$b_{i}$ & 50 & 50 & 50 \\
$v_{i}$ & 50 & 50 & 50 \\
part. coeff. & $61.5 \%$ & $25.6 \%$ & $12.8 \%$ \\
res. pr. lin. & -1.2 & 1.7 & 8.7 \\
res. pr. nonlin. & -1.5 & 2.0 & 8.9 \\
\hline
\end{tabular}

\begin{tabular}{|cccc|}
\hline Agent & 1 & 2 & 3 \\
\hline$\gamma_{i}$ & 2 & 5 & 10 \\
$b_{i}$ & 100 & 50 & 10 \\
$v_{i}$ & 50 & 50 & 50 \\
part. coeff. & $74.7 \%$ & $19.6 \%$ & $5.8 \%$ \\
res. pr. lin. & 1.9 & 2.4 & 18.6 \\
res. pr. nonlin. & 1.8 & 2.6 & 18.6 \\
\hline
\end{tabular}

\begin{tabular}{|cccc|}
\hline Agent & 1 & 2 & 3 \\
\hline$\gamma_{i}$ & 2 & 5 & 10 \\
$b_{i}$ & 50 & 50 & 50 \\
$v_{i}$ & 80 & 50 & 20 \\
part. coeff. & $70.7 \%$ & $21.8 \%$ & $7.5 \%$ \\
res. pr. lin. & -0.8 & 2.2 & 1.3 \\
res. pr. nonlin. & -0.9 & 2.4 & 1.4 \\
\hline
\end{tabular}

\begin{tabular}{|cccc|}
\hline Agent & 1 & 2 & 3 \\
\hline$\gamma_{i}$ & 2 & 5 & 10 \\
$b_{i}$ & 100 & 50 & 10 \\
$v_{i}$ & 80 & 50 & 20 \\
part. coeff. & $80.3 \%$ & $17.3 \%$ & $2.5 \%$ \\
res. pr. lin. & 1.0 & 2.6 & 5.5 \\
res. pr. nonlin. & 0.9 & 2.7 & 5.5 \\
\hline
\end{tabular}

The symbols $\gamma_{i}$ and $b_{i}$ refer to relative risk aversion and basis wealth respectively (see Table 1); $v_{i}$ denotes claim value. The last three rows in each panel indicate the participation coefficients in the linPEFF solution (i.e. the percentage of risk borne by each of the agents), the reservation prices of the linPEFF solution relative to autarky, and the reservation prices of the general (nonlinear) PEFF solution, also relative to autarky.

\section{CONCLUSIONS}

The main theoretical contributions of this paper are the introduction of the notion of linearly PEFF (linPEFF) solutions to risk sharing problems, and the proof of existence and uniqueness of such solutions for single-period problems under suitable conditions. Given the fact that in many situations we have both a unique linPEFF solution and a unique general (nonlinear) PEFF solution, it becomes of interest to investigate the relations between these solutions in particular situations. A few examples have been worked out.

Generally speaking, the benefits of risk sharing can be due both to a diversification effect and to a redistribution effect. The latter effect may even be present when agents are confronted with identical risks, and it arises from the fact that a risk sharing rule can modify the distributions of all of the participants, possibly in a way that is better for all. The particular cases that have been worked out show that a positive effect for all occurs sometimes, but not always. The results of the examples suggest that, compared to the redistribution effect, diversification is a stronger and more robust driver of risk sharing benefits. In the situations that we have considered, the agent who benefits most from pooling tends to be the one who is most risk averse.

Concerning the difference between linear and nonlinear rules of risk sharing, it appears that often the linear rule is quite competitive with the nonlinear rule. Since the linear rule is easier to compute and to implement, this observation might motivate the use of linPEFF rules. In situations in which particular levels are important to agents, as in the third example, the nonlinear rule may 
nevertheless have a psychological advantage, even if the difference with the linear rule is not large in utility terms.

\section{ACKNOWLEDGMENTS}

\section{I would like to thank Jaroslav Pazdera and Bas Werker for their comments on an earlier version of this paper. Comments by anonymous reviewers were also very helpful. The usual disclaimer applies.}

\section{NOTES}

1. Strictly speaking, these rules should be called "affine" rather than "linear", but we will stay with the entrenched terminology.

2. The notion of cautiousness was introduced by Wilson (1968). It is defined, for sufficiently differentiable utility functions, as the first derivative of the coefficient of absolute risk tolerance, which by itself is the inverse of the Arrow-Pratt coefficient of absolute risk aversion.

3. It may be argued that, if a liquid market exists in which the relevant risks can be traded freely, there would be little reason for collectives to form. As a result of this, the analysis of collectives is relevant in particular in situations in which such perfect market conditions are not present.

4. It should be taken into account that the argument taken by the utility function is the outcome to agent $i$ of the risk sharing agreement, while the agent may typically be expected to have other sources of income as well. Therefore, it is reasonable to include an offset representing basis wealth in the argument of the utility function. Examples are given in Section 4.

5. Positivity of the covariance for nondegenerate comonotonic variables follows from Höffding's covariance equality (Höffding, 1994).

6. The figure also serves to show some special features that may apply to the indifference price of the pooled risk as a function of the participation coefficient, such as a limited domain of definition (see (2.8)) and non-monotonicity.

7. The reservation price $c_{i}$ cannot be compared directly to the indifference price of total risk $\pi_{i}(X)$ that was discussed in Remark 3.3. The reservation price is a fixed amount that arises from the comparison of two discrete alternatives: participation in a pool with a given allocation rule, or autarky. The indifference price $\pi_{i}(X)$ is a price per unit of total risk; it presupposes participation in the pool, and it arises from comparison of alternatives that differ only infinitesimally.

8. The correlations as shown in the tables for the examples are the correlations between the lognormal risks, which differ slightly from the correlations $\rho$ appearing in Table 2 . The relation of the parameter $\rho$ to the correlation $\rho_{X}$ of the project outcomes is given by $\rho=\sigma^{-2} \log (1+$ $\left.\rho_{X}\left(\exp \left(\sigma^{2}\right)-1\right)\right)$.

9. Since in this paper, the risk $X$ is interpreted as an uncertain gain, rather than as an uncertain loss, there are sign differences with respect to the definition of the Esscher premium in the insurance literature. The quantity used here should perhaps be termed "Esscher value" rather than "Esscher premium".

\section{REFERENCES}

BAO, H., PONDS, E.H.M. and SCHUMACHER, J.M. (2017) Multi-period risk sharing under financial fairness. Insurance: Mathematics and Economics, 72, 49-66.

BARRIEU, P. and EL KAROUI, N. (2005) Inf-convolution of risk measures and optimal risk transfer. Finance and Stochastics, 9, 269-298.

BARrieu, P. and ScAndolo, G. (2008) General Pareto optimal allocations and applications to multi-period risks. ASTIN Bulletin, 38, 105-136. 
BIAGinI, S. and GuASONI, P. (2011) Relaxed utility maximization in complete markets. Mathematical Finance, 21, 703-722.

BORCH, K. (1962) Equilibrium in a reinsurance market. Econometrica, 30, 424- 444.

BRAmS, S.J. and TAYlor, A.D. (1996) Fair Division: From Cake-Cutting to Dispute Resolution. New York: Cambridge University Press.

BühlmanN, H. and JeWELL, W.S. (1979) Optimal risk exchanges. ASTIN Bulletin, 10, 243-262.

CARMOnA, R., ed. (2009) Indifference Pricing: Theory and Applications. Princeton, NJ: Princeton University Press.

Chateauneuf, A., DANA, R.-A. and TAllon, J.-M. (2000) Optimal risk-sharing rules and equilibria with Choquet-Expected-Utility. Journal of Mathematical Economics, 34, 191-214.

Chen, A., Pelsser, A. and Vellekoop, M. (2011) Modeling non-monotone risk aversion using SAHARA utility functions. Journal of Economic Theory, 146, 2075-2092.

Chen, D. and BeEtSMA, R. (2015) Mandatory participation in occupational pension schemes in the Netherlands and other countries: An update. Technical Report DP 10/2015-032, Netspar.

DAI, R. and SCHUMACHER, J.M. (2009) Welfare analysis of conditional indexation schemes from a two-reference-point perspective. Journal of Pension Economics and Finance, 8, 321-350.

FiliPOVIC, D. and SvindLAND, G. (2008) Optimal capital and risk allocations for law- and cashinvariant convex functions. Finance and Stochastics, 12, 423-439.

Gale, D. (1977) Fair division of a random harvest. Technical Report ORC 77-21, Operations Research Center, UC Berkeley.

Gale, D. and Sobel, J. (1979) Fair division of a random harvest: The finite case. In General Equilibrium, Growth, and Trade. Essays in Honor of Lionel McKenzie (eds. J.R. Green and J. Scheinkman), pp. 193-198. New York: Academic Press.

HöfFDING, W. (1994) Scale-invariant correlation theory. In The Collected Works of Wassily Hoeffding (eds. N.I. Fisher and P.K. Sen), pp. 57-108. New York: Springer.

Homma, T. (1952) A theorem on continuous functions. Kodai Mathematical Seminar Reports, 4 , $13-16$.

HuANG, C.-F. and Litzenberger, R. (1985) On the necessary condition for linear sharing and separation: A note. Journal of Financial and Quantitative Analysis, 20, 381-384.

Jouini, E., Schachermayer, W. and TouZI, N. (2008) Optimal risk sharing for law invariant monetary utility functions. Mathematical Finance, 18, 269-292.

KAMPS, U. (1998) On a class of premium principles including the Esscher principle. Scandinavian Actuarial Journal, 1998(1), 75-80.

KRAMKOV, D. and SCHACHERMAYER, W. (1999) The asymptotic elasticity of utility functions and optimal investment in incomplete markets. Annals of Applied Probability, 9, 904-950.

MARIĆ, V. and TOMIĆ, M. (1990) A classification of solutions of second order linear differential equations by means of regularly varying functions. Publications de l'Institut Mathématique, 48, 199-207.

MolenAAR, R.D.J. and PONDS, E.H.M. (2012/13) Risk sharing and individual lifecycle investing in funded collective pensions. Journal of Risk, 15(2), 103-124.

PAK, I. (2010) Lectures on Discrete and Polyhedral Geometry. http://www.math.ucla.edu/ $\sim$ pak/geompol8.pdf.

PAZDERA, J., SCHUMACHER, J.M. and WERKER, B.J.M. (2017) The composite iteration algorithm for finding efficient and financially fair risk-sharing rules. Journal of Mathematical Economics, 72, 122-133.

RotAR, V.I. (2007) Actuarial Models. The Mathematics of Insurance. Boca Raton: Chapman \& Hall/CRC.

SEAL, H.L. (1969) Stochastic Theory of a Risk Business. New York: Wiley.

TUCKER, A. (1995) The parallel climbers puzzle. Math Horizons, 3(2), 22-24.

van Heerwaarden, A.E., KaAs, R. and Goovaerts, M.J. (1989) Properties of the Esscher premium calculation principle. Insurance: Mathematics and Economics, 8, 261-267.

WhitTAKer, J.V. (1966) A mountain-climbing problem. Canadian Journal of Mathematics, 18, 873-882.

WILSON, R. (1968) The theory of syndicates. Econometrica, 36, 119-132.

WUERTH, A.M. and SCHUMACHER, J.M. (2011) Risk aversion for nonsmooth utility functions. Journal of Mathematical Economics, 47, 109-128. 
JOHANNES M. SCHUMACHER (Corresponding author)

University of Amsterdam

Faculty of Economics and Business

Section Quantitative Economics

Roetersstraat 11, 1018 WB Amsterdam

The Netherlands

E-Mail: j.m.schumacher@uva.nl

\section{APPENDIX}

\section{A.1. Proof of Theorem 3.1}

For brevity, write $s_{i}=E\left[X u_{i}^{\prime}\left(a_{i} X+b_{i}\right)\right]$ and $t_{i}=E\left[u_{i}^{\prime}\left(a_{i} X+b_{i}\right)\right]$. Note that $t_{i}>0$ for all $i$. We need to show that the following conditions are equivalent: (i) there exist $\alpha_{i}>0, \lambda$, and $\mu$ such that $\alpha_{i} s_{i}=\lambda$ and $\alpha_{i} t_{i}=\mu$ for all $i$; (ii) there is a constant $c$ such that $s_{i} / t_{i}=c$ for all $i$. If (i) holds, then the constant $\mu$ must be positive, and (ii) is satisfied with $c=\lambda / \mu$. Conversely, if (ii) holds, then in (i) we can take $\alpha_{i}=1 / t_{i}, \lambda=c$, and $\mu=1$.

\section{A.2. Connection to the Borch criterion}

The aim of this section is to provide an intuitive understanding of the relation between Borch's well-known criterion for Pareto efficiency on the one hand, and on the other hand the criterion for Pareto efficiency within the class of linear allocation rules as given in Theorem 3.1. For this purpose, introduce the class of conditionally linear allocation rules, which is defined as follows. Suppose that the range of possible values of total risk $X$ has been subdivided into a finite number of non-overlapping intervals $A_{k}, k=1, \ldots, N$. An allocation rule is said to be conditionally linear (with respect to these sets) if there exist constants $a_{i}^{k}$ and $b_{i}^{k}(i=1, \ldots, n$, $k=1, \ldots, N)$ with

$$
\sum_{i=1}^{n} a_{i}^{k}=1, \quad \sum_{i=1}^{n} b_{i}^{k}=0 \quad(k=1, \ldots, N),
$$

such that the claim allocated to agent $i$ is given by

$$
y_{i}(x)=\sum_{k=1}^{N} \mathbb{1}_{A_{k}}(x)\left(a_{i}^{k} x+b_{i}^{k}\right) .
$$

Since the set of allocation functions defined in this way is convex, the set of Pareto efficient solutions can be determined by optimizing a weighted sum of agents' preference functions subject to the feasibility constraints. Using weights $\alpha_{i}$, the first-order conditions for optimality are that there should exist constants $\lambda_{k}$ and $\mu_{k}(k=1, \ldots, N)$ such that

$$
\begin{array}{r}
\alpha_{i} E\left[X u_{i}^{\prime}\left(Y_{i}\right) \mid X \in A_{k}\right]=\lambda_{k}, \\
\alpha_{i} E\left[u_{i}^{\prime}\left(Y_{i}\right) \mid X \in A_{k}\right]=\mu_{k},
\end{array}
$$


for $i=1, \ldots, n$ and $k=1, \ldots, N$. These two conditions may be replaced by the second condition together with the requirement that there are constants $c_{k}$ such that

$$
\frac{E\left[X u_{i}^{\prime}\left(Y_{i}\right) \mid X \in A_{k}\right]}{E\left[u_{i}^{\prime}\left(Y_{i}\right) \mid X \in A_{k}\right]}=c_{k},
$$

for all $i$ and $k$. This condition expresses that an allocation-induced adjusted conditional expectation of the pooled risk $X$ should be the same for all agents.

Heuristically, Borch's rule can be derived from this by considering what happens when the sets $A_{k}$ are reduced to singletons. The index $k$ may then be replaced by the argument $x$, and we write $\lambda(x), \mu(x)$, and $c(x)$ instead of $\lambda_{k}, \mu_{k}$, and $c_{k}$. Since, we are conditioning on the outcome of $X$, the expectation symbols in (A1) and (A2) can be dropped, and the two conditions become

$$
\begin{aligned}
\alpha_{i} x u_{i}^{\prime}\left(y_{i}(x)\right) & =\lambda(x), \\
\alpha_{i} u_{i}^{\prime}\left(y_{i}(x)\right) & =\mu(x) .
\end{aligned}
$$

The first condition is implied by the second one (take $\lambda(x)=x \mu(x)$ ), so that only the second condition needs to be retained. This is Borch's condition. Therefore, the classical result of Borch can be viewed as a limit case of the efficiency condition for conditionally linear allocation rules when the number $N$ of sets $A_{k}$ tends to infinity. In contrast, Theorem 3.1 of this paper is concerned with the case $N=1$. In this situation, it is condition (A2) which becomes redundant. In this way, Borch's condition and the condition of Theorem 3.1 can be viewed as representing opposite ends of a range of efficiency conditions. At both extremes, the general conditions simplify, albeit in different ways.

\section{A.3. Properties of the generalized Esscher transform}

To support the analysis in Section 3, in this section some properties are derived of the mapping

$$
g(a)=E\left[X u^{\prime}(a X)\right] / E\left[u^{\prime}(a X)\right],
$$

where $u$ is a utility function of the type described in Assumption 2.2. The analysis applies to functions occurring in the condition (3.9) for linPEFF solutions after a reparametrization. In the special case $u(x)=-e^{-x}$, the right-hand side of (A4) is known as the Esscher premium with parameter $a$ corresponding to the risk $X .{ }^{9}$ Generalized Esscher premium principles of the form $E[X h(X)] / E[h(X)]$ were studied for instance by Kamps (1998).

The random variable $u^{\prime}(a X) / E\left[u^{\prime}(a X)\right]$ is positive, and its expectation is 1 ; therefore, it can be taken as a Radon-Nikodym derivative that defines a change of measure from $P$ to a new measure $P^{a}$. Corresponding operators, such as expectation and covariance, will be indicated by superscript $a$. In particular, we write

$$
E^{a}[X]=\frac{E\left[X u^{\prime}(a X)\right]}{E\left[u^{\prime}(a X)\right]}=g(a) .
$$

The Arrow-Pratt coefficient of risk aversion associated to the utility function $u(x)$ is denoted by $r(x)$. The assumptions on the random variable $X$ in the lemma below correspond via reparametrization to the application in the main text. 
Lemma A.1. Let $X$ be a discrete random variable with finitely many possible values, satisfying $\min X<0$. Let $u$ be a utility function that satisfies the conditions of Assumption 2.2, with $L<0$ where $L$ denotes the left boundary of the domain of $u$. Define

$$
\bar{a}= \begin{cases}L /(\min X) & \text { if } L \text { is finite } \\ \infty & \text { if } L=-\infty\end{cases}
$$

The following properties hold.

i. The function $g(a)=E^{a}[X]$ is continuous on the interval $[0, \bar{a})$.

ii. We have $E^{0}[X]=E[X]$, and $E^{a}[X]<E[X]$ for $a>0$.

iii. If $L$ is finite, then

$$
\lim _{a \rightarrow \bar{a}} E^{a}[X]=\min X .
$$

If $L=-\infty$, then the statement ( A6) holds under the asymptotic condition (2.3).

iv. The function $g(a)=E^{a}[X]$ is differentiable on the interval $(0, \bar{a})$, and its derivative is given by

$$
\frac{d E^{a}[X]}{d a}=-\operatorname{Cov}^{a}(X, X r(a X)) .
$$

Proof. Continuity, differentiability, and the relation $E^{0}[X]=E[X]$ are verified immediately. Since the marginal utility $u^{\prime}$ is strictly decreasing, the variables $X$ and $u^{\prime}(a X)$ are countermonotonic for any fixed nonnegative value of $a$. Given also that $X$ is nondegenerate, the inequality $\operatorname{Cov}\left(X, u^{\prime}(a X)\right)<0$ holds for $a>0$, which implies (ii). Let $x_{1}=\min X<$ $x_{2}<\cdots<x_{N}$ denote the possible values of $X$. By the definition of the number $\bar{a}$, condition (iii) in Assumption 2.2, and the assumption $\min X<0$, we have

$$
\lim _{a \rightarrow \bar{a}} u^{\prime}\left(a x_{1}\right)=\infty .
$$

If $L>-\infty$, then $\lim _{a \rightarrow \bar{a}} u^{\prime}\left(a x_{k}\right)=u^{\prime}\left(\left(x_{k} / x_{1}\right) L\right)$ for $k \geq 2$ with $x_{k}<0$, and $\lim _{a \rightarrow \bar{a}} u^{\prime}\left(a x_{k}\right) \leq$ $u^{\prime}(0)$ for $k$ with $x_{k}>0$, so that

$$
\lim _{a \rightarrow \bar{a}} \frac{u^{\prime}\left(a x_{k}\right)}{u^{\prime}\left(a x_{1}\right)}=0 \quad \text { for all } k \geq 2
$$

This implies (A6). In the case in which $L=-\infty$ and the assumption (2.3) is satisfied, the relation (A8) follows from the proof of Lemma 3 in Marić and Tomić (1990). Finally, the derivative $g^{\prime}(a)$ can be calculated as follows:

$$
\begin{aligned}
\frac{d E^{a}[X]}{d a} & =\frac{d}{d a} \frac{E\left[X u^{\prime}(a X)\right]}{E\left[u^{\prime}(a X)\right]} \\
& =\frac{E\left[X^{2} u^{\prime \prime}(a X)\right]}{E\left[u^{\prime}(a X)\right]}-\frac{E\left[X u^{\prime \prime}(a X)\right]}{\left(E\left[u^{\prime}(a X)\right]\right)^{2}} E\left[X u^{\prime}(a X)\right] \\
& =-\frac{E\left[X^{2} r(a X) u^{\prime}(a X)\right]}{E\left[u^{\prime}(a X)\right]}+\frac{E\left[X r(a X) u^{\prime}(a X)\right]}{E\left[u^{\prime}(a X)\right]} \frac{E\left[X u^{\prime}(a X)\right]}{E\left[u^{\prime}(a X)\right]} \\
& =-E^{a}\left[X^{2} r(a X)\right]+E^{a}[X r(a X)] E^{a}[X] \\
& =-\operatorname{Cov}^{a}(X, X r(a X)) .
\end{aligned}
$$


The lemma above contains extensions of results that were obtained for the case $u(x)=$ $-e^{-x}$ (the Esscher principle) by van Heerwaarden et al. (1989). In particular, the formula (A7) is a generalized version of Equation (4) in the cited paper.

Remark A.2. The quantity $\lim _{x \rightarrow-\infty}-x r(x)$ may be referred to as the asymptotic coefficient of relative risk aversion for large losses. The reader may wonder what can be said about the limit $\lim _{a \rightarrow \infty} E^{a}[X]$ when this asymptotic coefficient is finite, rather than infinite as assumed in the lemma above. Using techniques as in Marić and Tomic (1990), one can calculate that

$$
\lim _{a \rightarrow \infty} E^{a}[X]=\frac{E[X h(X)]}{E[h(X)]}
$$

where

$$
h(x)=\left\{\begin{array}{cc}
(-x)^{\gamma} & (x<0) \\
0 & (x \geq 0),
\end{array}\right.
$$

and $\gamma=\lim _{x \rightarrow-\infty}-x r(x)$. If the coefficient of risk aversion tends to zero faster than $1 /|x|$ as $x$ tends to $-\infty$, so that the asymptotic coefficient of relative risk aversion for large losses is equal to zero, then $\lim _{a \rightarrow \infty} E^{a}[X]=E[X \mid X<0]$. This is a Tail-Value-at-Risk (expected value of the outcome conditional on exceedance of a certain level toward the adverse side).

\section{A.4. Proof of Theorem 3.7}

For the proof of Theorem 3.7, we make use of a result that is known as the "mountain climbing theorem" (Homma, 1952; Whittaker, 1966). The name is derived from the interpretation of the theorem as a positive answer to the question whether it is possible for two climbers, both starting at sea level and climbing different sides of a mountain, to reach the top while coordinating their movements in such a way that at all times they are at the same altitude. We need in fact a version with $n$ climbers rather than two, but this requires only a straightforward modification of the graph-theoretic proof as given by Tucker (1995); see also Pak (2010, Theorem 5.5, Exc. 5.24). The theorem can be stated as follows.

Lemma A.3. Let $n$ continuous and piecewise monotonic functions $f_{i}$ from $[0,1]$ to $[0,1]$ be given, with $f_{i}(0)=0$ and $f_{i}(1)=1$ for all $i$. Then, there exist continuous functions $g_{i}$ from $[0,1]$ to $[0,1]$, with $g_{i}(0)=0$ and $g_{i}(1)=1$ for all $i=1, \ldots, n$, such that $f_{1}\left(g_{1}(x)\right)=$ $f_{2}\left(g_{2}(x)\right)=\cdots=f_{n}\left(g_{n}(x)\right)$ for all $0 \leq x \leq 1$.

Theorem 3.7 may now be proven as follows. Assume first that the domains of the utility functions of all agents are bounded below. In this case, all upper bounds $\bar{a}_{i}$ are finite. It follows from Lemma A.1 that for each $i$ we can define a continuous function $f_{i}$ from $[0,1]$ to $[0,1]$ by

$$
f_{i}(t)=\left\{\begin{array}{cl}
\frac{E[X]-E_{i}^{\bar{a}_{i} t}[X]}{E[X]-\min X} & \text { for } 0 \leq t<1 \\
1 & \text { for } t=1 .
\end{array}\right.
$$

To these functions, we can apply the mountain climbing theorem. We then obtain continuous functions $g_{i}$ with $g_{i}(0)=0$ and $g_{i}(1)=1$ such that

$$
E_{1}^{\bar{a}_{1} g_{1}(t)}[X]=\cdots=E_{n}^{\bar{a}_{n} g_{n}(t)}[X] \quad \text { for all } 0 \leq t \leq 1 .
$$

Define

$$
h(t)=\sum_{i=1}^{n} \bar{a}_{i} g_{i}(t)
$$


The function $h(t)$ is continuous; moreover, it satisfies $h(0)=0$ and $h(1)=\sum_{i=1}^{n} \bar{a}_{i}>1$ (see Lemma 2.8). Therefore, there exists $t^{*} \in(0,1)$ such that $h\left(t^{*}\right)=1$. A solution to Problem 2.9 is now obtained by setting $a_{i}=\bar{a}_{i} g_{i}\left(t^{*}\right)$ for $i=1, \ldots, n$.

In the case in which not all lower bounds $L_{i}$ are finite and condition (2.3) applies, we know by Lemma A.1 that $E_{i}^{a_{i}}[X]$ converges to $\min X$ as $a_{i}$ tends to infinity for agents $i$ such that $L_{i}=-\infty$. One can then define a corresponding continuous function $f_{i}$ from $[0,1]$ to $[0,1]$, as in the argument above, this time using a suitable continuous transformation which maps the interval $[0, \infty]$ to $[0,1]$, such as $\frac{2}{\pi} \arctan x$. The proof then follows by the same reasoning as above.

If it is assumed that all functions $a_{i} \mapsto E_{i}^{a_{i}}[X]$ are strictly monotonic, then for each value of $c$ there can be at most one solution $a_{i}$ to the Equation (3.9). The functions $a_{i}(c)$ implicitly defined by (3.9) are strictly increasing as $c$ is decreased from its maximal value $E[X]$, and they converge to $\sum_{i=1}^{n} \bar{a}_{i}>1$. Consequently, there exists a uniquely determined value $c^{*}$ at which $\sum_{i=1}^{n} a_{i}\left(c^{*}\right)=1$. The vector $\left(a_{1}\left(c^{*}\right), \ldots, a_{n}\left(c^{*}\right)\right)$ then defines the unique solution to Problem 2.9. This completes the proof.

Remark A.4. The condition of strict monotonicity of the functions $a_{i} \mapsto E_{i}^{a_{i}}[X]$ can be weakened to strict monotonicity of these functions on the interval $\left[0, \min \left(\bar{a}_{i}, 1\right)\right)$.

Remark A.5. An additional case in which existence of solution can be proven, without the condition (2.3), is the following. Suppose there exist a constant $\bar{a}>1$ and an agent $i$ with $\bar{a}_{i} \geq \bar{a}$ such that $E_{i}^{a_{i}}[X]>E_{i}^{\bar{a}}[X]$ for all $0 \leq a_{i}<\bar{a}$, and $E_{i}^{\bar{a}}[X] \geq E_{j}^{\bar{a}}[X]$ for all agents $j$ with $\bar{a}_{j} \geq \bar{a}$. Without loss of generality, let the index of the agent appearing in the condition be 1. For $i=1, \ldots, n$, define

$$
\tilde{a}_{i}=\min \left\{a_{i} \mid E_{i}^{a_{i}}[X]=E_{1}^{\bar{a}}[X]\right\} .
$$

Note that $\sum_{i=1}^{n} \tilde{a}_{i}>1$. The argument in the case of $L_{i}>-\infty$ for all $i$ can now be followed with the constants $\tilde{a}_{i}$ instead of $\bar{a}_{i}$.

\section{A.5. Proof of Corollary 3.8}

It follows from item (iv) of Lemma A.1 that

$$
\frac{d E_{i}^{a_{i}}[X]}{d a_{i}}=-\operatorname{Cov}^{a_{i}}\left(X-v,(X-v) r_{i}\left(a_{i}(X-v)+v_{i}\right)\right),
$$

where the superscript $a_{i}$ refers to the measure that has Radon-Nikodym derivative $u_{i}^{\prime}\left(a_{i}(X-\right.$ $\left.v)+v_{i}\right) / E\left[u_{i}^{\prime}\left(a_{i}(X-v)+v_{i}\right)\right]$ with respect to $P$. Under the condition stated in the corollary, the variables $X-v$ and $(X-v) r_{i}\left(a_{i}(X-v)+v_{i}\right)$ are comonotonic, which implies that the righthand side in the expression above is negative. Consequently, the quantity $E_{i}^{a_{i}}[X]$ is strictly monotonically decreasing in $a_{i}$, and the statement follows from Theorem 3.7. 